\title{
Activation of a Lotus japonicus Subtilase Gene During Arbuscular Mycorrhiza Is Dependent on the Common Symbiosis Genes and Two cis-Active Promoter Regions
}

\author{
Naoya Takeda, ${ }^{1}$ Kristina Haage, ${ }^{1}$ Shusei Sato, ${ }^{2}$ Satoshi Tabata, ${ }^{2}$ and Martin Parniske ${ }^{1}$ \\ ${ }^{1}$ University of Munich (LMU), Biology, Genetics, Großhaderner Str. 2-4, 82152 Martinsried, Germany; ${ }^{2}$ Kazusa DNA Research \\ Institute, 2-6-7 Kazusa-kamatari, Kisarazu, Chiba 292-0818, Japan
}

Submitted 27 September 2010. Accepted 14 January 2011.

\begin{abstract}
The subtilisin-like serine protease $S b t M 1$ is strongly and specifically induced during arbuscular mycorrhiza (AM) symbiosis in Lotus japonicus. Another subtilase gene, SbtS, is induced during early stages of nodulation and AM. Transcript profiling in plant symbiosis mutants revealed that the AM-induced expression of SbtM1 and the gene family members SbtM3 and SbtM4 is dependent on the common symbiosis pathway, whereas an independent pathway contributes to the activation of $S b t S$. We used the specific spatial expression patterns of $S b t M 1$ promoter $\beta$-D-glucuronidase (GUS) fusions to isolate cis elements that confer AM responsiveness. A promoter deletion and substitution analysis defined two cis regions (region I and II) in the SbtMI promoter necessary for AM-induced GUS activity. $35 \mathrm{~S}$ minimal promoter fusions revealed that either of the two regions is sufficient for $A M$ responsiveness when tested in tandem repeat arrangement. Sequence-related regions were found in the promoters of AM-induced subtilase genes in Medicago truncatula and rice, consistent with an ancient origin of these elements predating the divergence of the angiosperms.
\end{abstract}

Arbuscular mycorrhiza (AM) is a mutualistic interaction between terrestrial plants and Glomeromycota fungi (Schüssler et al. 2001). The network of AM fungal hyphae facilitates uptake of water and nutrients, predominantly phosphate and nitrogen, from the soil, which are provided to the plant host. In return, the fungus obtains photosynthetic products from the plant (Bago 2003; Parniske 2008). The development of the symbiotic interaction involves a specific exchange of signaling molecules and subsequent formation of symbiotic structures in the host root (Harrison 2005). Hyphae of AM fungi perceive strigolactones, signaling molecules from the host, which activate hyphal branching, elongation, and metabolism (Akiyama et al. 2005; Besserer et al. 2006). AM fungi produce diffusible signaling molecules called Myc factors. Myc factors induce plant gene expression (Kosuta et al. 2003; Mukherjee and Ane 2011)

Current address of N. Takeda: Division of Symbiotic Systems, National Institute for Basic Biology, Nishigonaka 38, Myodaiji, Okazaki, Aichi, 444-8585, Japan.

Corresponding author: M. Parniske; E-mail parniske@lmu.de; Fax:+49 89-2180-74702.

* The $\boldsymbol{e}$-Xtra logo stands for "electronic extra" and indicates that seven supplementary figures and one supplementary table are published online. and trigger characteristic nuclear calcium spiking (Chabaud et al. 2011; Kosuta et al. 2008) in the host plant. AM fungal hyphae contact epidermal cells and are guided into the host root (Genre et al. 2005, 2008). The intraradical hyphae elongate in intercellular spaces of cortical cells along the stele (Demchenko et al. 2004). The symbiotic structures, the arbuscules, are formed in cortical cells (Parniske 2008). This process is associated with the induction of genes involved in arbuscule function, and lysophosphatidylcholine has been implicated in the induction of AM-specific phosphate transporter genes (Drissner et al. 2007). The elaborate membrane structures in arbuscules enlarge the interface between the host plant and AM fungi, which facilitates nutrient exchange (Bonfante and Perotto 1995; Gianinazzi-Pearson 1996).

In order to investigate the molecular principles underlying the AM symbiosis, transcriptome analysis in several independent laboratories has identified AM-induced genes from monocots and dicots (Guether et al. 2009; Guimil et al. 2005; Gutjahr et al. 2008; Kistner et al. 2005; Liu et al. 2003). The genetic analysis of symbiotic mutants has identified a core set of genes required for early developmental steps of both AM and the root nodule symbiosis (RNS) of legumes. The model legumes Lotus japonicus and Medicago truncatula have been instrumental in the isolation of a set of eight genes-SYMRK (DMI2), CASTOR, POLLUX (DMI1), NUP85, NUP133, SEH1, CCAMK (DMI3), and CYCLOPS (IPD3) (Ané et al. 2004; Endre et al. 2002; Groth et al. 2010; Imaizumi-Anraku et al. 2005; Levy et al. 2004; Stracke et al. 2002; Tirichine et al. 2006a; Yano et al. 2008) — that are implicated in a common symbiosis regulatory network (Kistner and Parniske 2002; Markmann et al. 2008; Oldroyd and Downie 2008). This common symbiosis pathway appears to be functionally conserved between legumes and rice (Banba et al. 2008; Chen et al. 2007, 2008; Gutjahr et al. 2008; Markmann et al. 2008), suggesting an ancient role in symbiosis that dates back at least to the common ancestor of the angiosperms (Markmann and Parniske 2009). In addition to the common symbiosis network, genetic components specifically required for the root nodule symbiosis have been identified (Oldroyd and Downie 2008). Comparatively little is known about AM symbiosis-specific components and signaling factors (Drissner et al. 2007; Javot et al. 2007; Kuhn et al. 2010; Maeda et al. 2006; Zhang et al. 2010). Reverse genetic approaches successfully demonstrated essential roles of several genes that are specifically induced during AM (Floss et al. 2008; Javot et al. 2007; Maeda et al. 2006; Takeda et al. 2009). 
We performed large-scale expression analysis and isolated AM-induced genes in L. japonicus (Kistner et al. 2005). In particular, genes encoding proteases of the subtilisin-like serine protease family (subtilase) (Rawlings and Barrett 1999; Siezen and Leunissen 1997) were highly and specifically induced during AM (Kistner et al. 2005; Takeda et al. 2009). The AMinduced subtilase genes $S b t M 1, S b t M 3, S b t M 4$, and $S b t S$ were differentially regulated during AM and RNS (Takeda et al. 2007). Functional analysis using RNAi revealed that SbtM1 and SbtM3 are required for the development of the AM symbiosis, in particular of the arbuscules (Takeda et al. 2009). Because SbtM1 and SbtM3 carry predicted signal peptides at their amino termini, and the SbtM1 signal peptide targeted Venus, a fluorescent marker protein, to the intercellular and peri-arbuscular spaces of the host cell, we hypothesize that SbtM1 and SbtM3 cleave a substrate located at the plant-fungus interface and that this cleavage is crucial for the formation of arbuscules (Takeda et al. 2009).

The regulation of SbtMI is highly specific, because the gene is only expressed during AM (Kistner et al. 2005). Therefore, its promoter and the responsible cis elements are useful tools for the analysis of AM-signaling mechanisms. The aim of the present study was a refined analysis of the genetic regulation of AM. For this, we analyzed the expression of SbtM1 and other symbiotic marker genes in the context of symbiotic mutants. This analysis confirmed the existence of AM-specific signaling pathways. As a first step toward the identification of AM-specific regulatory components, we determined AMresponsive cis regions in the $S b t M 1$ promoter using $S b t M 1$ promoter: $\beta$-D-glucuronidase (GUS) fusions. The spatial GUS staining pattern during AM in roots transformed with SbtM1 promoter:GUS fusions provided a reliable readout for the promoter's AM responsiveness. Using this approach, we identified two cis regions and showed that both of them independently confer AM responsiveness. These cis regions provide an important tool for the identification and analysis of regulatory components specific for AM.

\section{RESULTS}

AM-induced gene expression in symbiotic plant mutants.

We analyzed AM-induced gene expression in roots of symbiotic mutants of $L$. japonicus by quantitative real-time reverse-transcription polymerase chain reaction (qRT-PCR) (Fig. 1). In wild-type (WT) roots inoculated with the AM fungus Glomus intraradices, a clear induction of the AM-induced genes SbtM1, SbtM3, SbtM4, and SbtS was observed but no induction of the subtilase pseudogenes SbtM2 and SbtM5 (Fig. 1A). The phosphate transporter gene PT4 of M. truncatula is exclusively expressed in arbuscule-containing cells (Harrison et al. 2002), and we observed PT4 of L. japonicus to be induced in WT roots (Fig. 1A), thus confirming earlier observations by Takeda and associates (2009). In inoculated Nod factor receptor (nfr) mutants nfrl-1 (sym1-1) and nfr5-2 (sym5-2), AMinduced genes were expressed at the same level as in the WT control (Fig. 1A), consistent with the fact that AM symbiosis is unaffected in these mutants (Wegel et al. 1998). Seven common sym mutants-symrk-10, castor-2 (sym4-2), pollux-1 (sym23-1), nup133-1 (sym3-1), nup85-1 (sym24), ccamk-2 (sym15-2), and cyclops-3 - that are defective at early steps of AM development (Kistner et al. 2005) showed no induction or lower expression of SbtM1, SbtM3, SbtM4, and PT4 compared with noninoculated WT roots (Fig. 1A). These induction patterns were in line with the previously observed lack of SbtMI expression in common sym mutants (Kistner et al. 2005). The lower basal expression of AM-induced genes in common sym mutants is perhaps caused by a loss of background signaling.

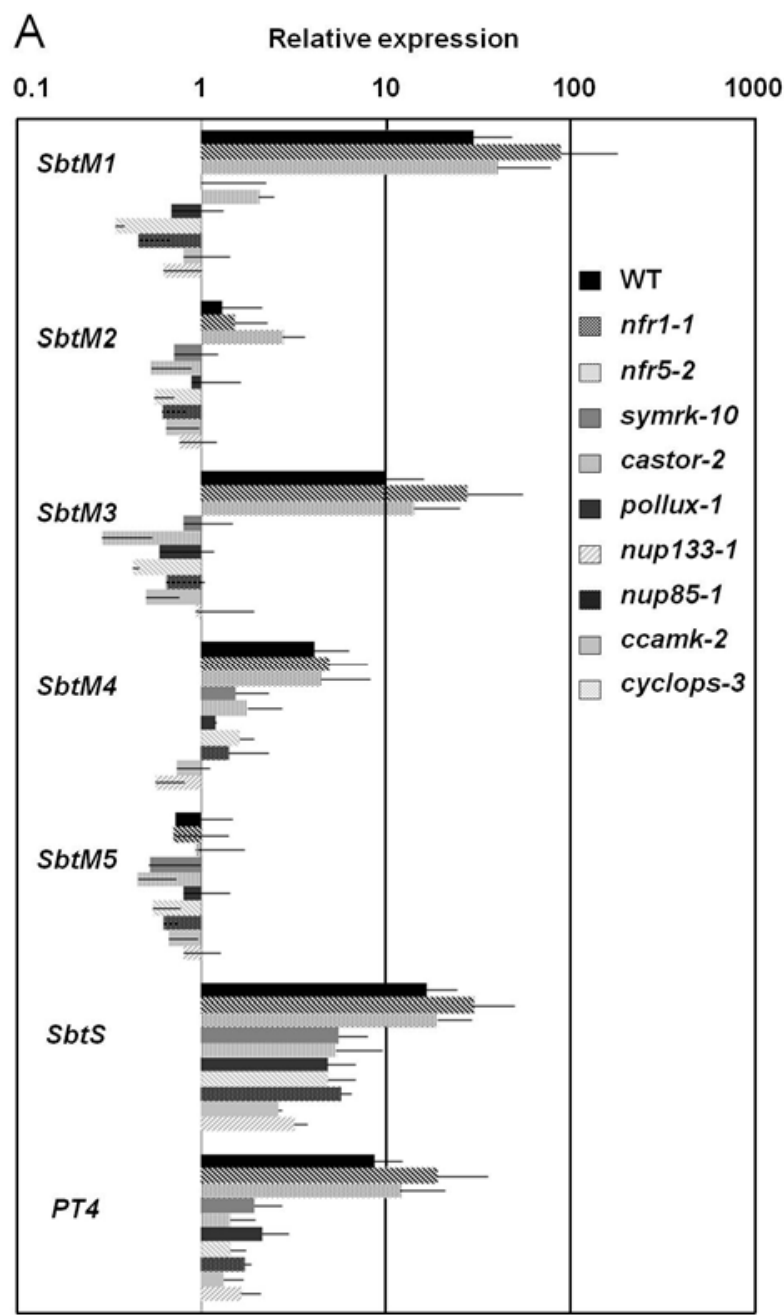

B

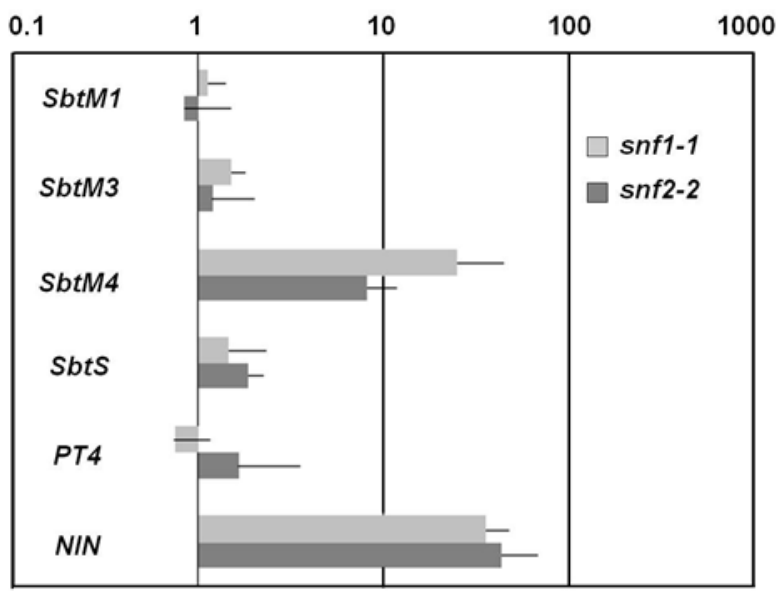

Fig. 1. Gene expression in symbiotic mutants. A, Expression of arbuscular mycorrhiza (AM)-induced genes in Lotus japonicus (Gifu) wild-type (WT) and mutant roots was analyzed by quantitative real-time reverse-transcription polymerase chain reaction. Plants were cocultivated with Glomus intraradices for 2 weeks in chive nurse pots. Values represent relative expression compared with noninoculated WT roots (relative expression $=1$ ). B, Expression of SbtM1, SbtM3, SbtM4, SbtS, PT4, and NIN in snf1-1 and snf2-2 mutant roots were compared with noninoculated WT roots (relative expression = 1). Activation of SbtM4 and NIN was observed in snf1-1 and $s n f 2-2$ but no obvious induction of $S b t M 1, S b t M 3$, and $S b t S$ in roots 4 weeks after germination. Total RNA was extracted from four to eight roots. Mean values of the relative expressions of three to four independent experiments are displayed in logarithmic scale. Error bars represent standard deviation ( $n=3$ to 4$)$. 
SbtS induction was still observed in common sym mutants, where the expression level was reduced to one-fourth to onethird compared with WT roots. This result revealed the existence of a symbiotic signaling pathway that activates $S b t S$ expression independent of the common SYM pathway.

We also analyzed the expression of AM-induced genes in spontaneous nodule formation (snf) mutants (Tirichine et al. 2006b). Two snf mutants—snfl, encoding a deregulated version of CCaMK (Tirichine et al. 2006a) and snf2, expressing a constitutively activated cytokinin receptor (Tirichine et al. 2007) form spontaneous root nodules and induce downstream nodulation-related marker genes such as NIN in the absence of rhizobia (Tirichine et al. 2006b). Because CCaMK is also required
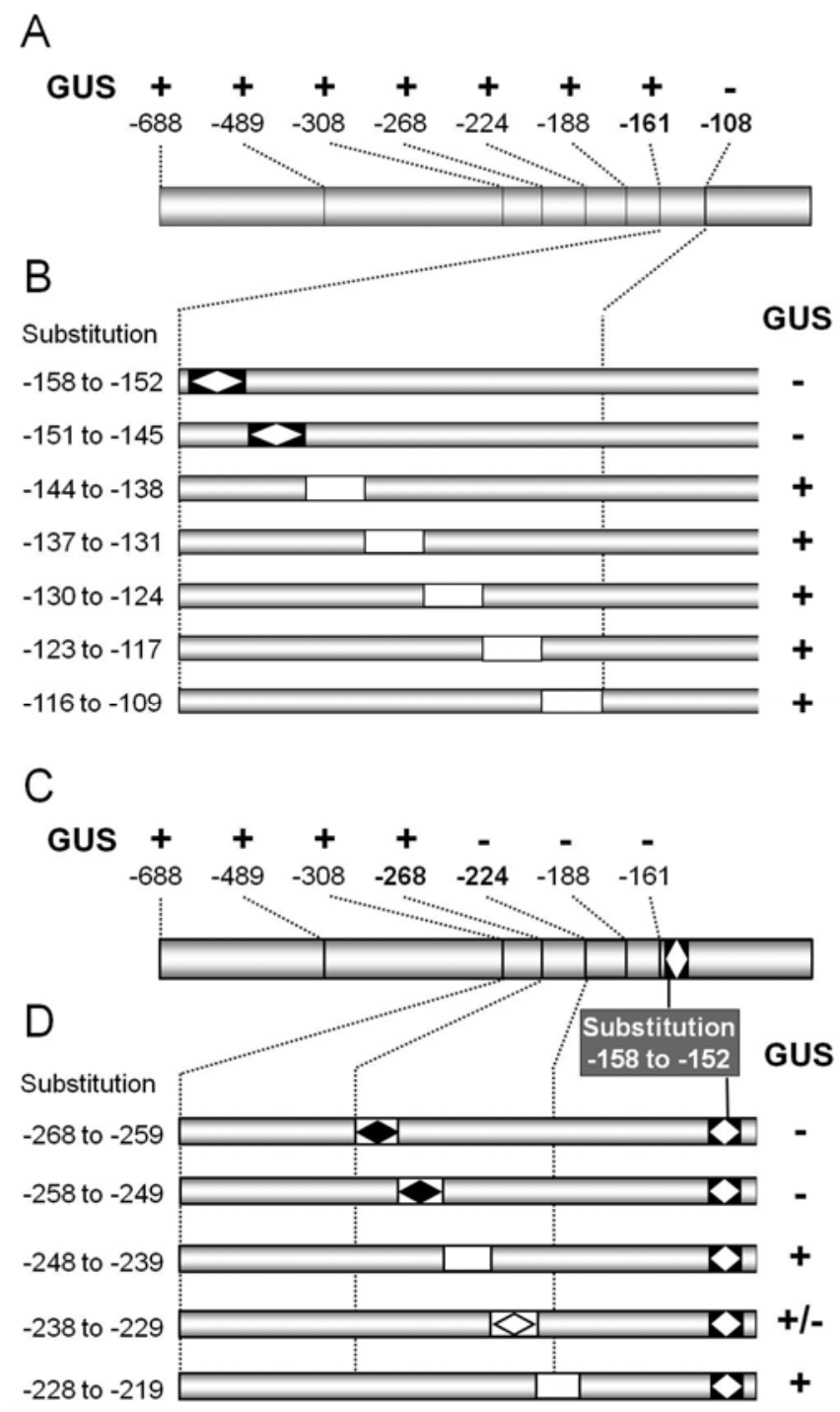

Fig. 2. Deletion and substitution analysis of the $S b t M 1$ promoter. A series of SbtM1 promoter deletions and substitutions was fused to the $\beta$ glucuronidase $(G U S)$ reporter gene and the fusion constructs were transformed in Lotus japonicus hairy roots. Transgenic roots were stained to analyze GUS activity 1 and 2 weeks after inoculation with Glomus intraradices. Arbuscular mycorrhiza (AM)-induced GUS expression was scored $(+)$ if patchy staining along the root and strong staining in arbuscule containing cells were observed. Numbers indicate the nucleotide position relative to the A of the SbtM1 translation start codon. $\mathbf{B}$ and $\mathbf{D}$, Boxes represent substituted (A/C or $\mathrm{T} / \mathrm{G})$ regions. A, Deletion analysis of the SbtM1 promoter. B, Substitution analysis of the SbtM1 promoter between -158 and -109 bp. C, Deletion analysis of the SbtM1 promoter containing a substitution between -158 and -152 bp. D, Substitution analysis between -268 and -219 bp of the SbtM1 promoter containing a substitution between -158 and $-152 \mathrm{bp}$. for AM, we investigated whether these mutants also activate AM-related marker genes spontaneously. The snfl-1 and snf22 mutants formed spontaneous nodules 4 weeks after germination and showed elevated NIN expression (Fig. 1B). Among the AM-marker genes, only SbtM4, which is induced in AM and RNS, showed increased transcript abundance in both mutants. However, the other AM-marker genes (SbtM1, SbtM3, SbtS, and PT4) were not induced, indicating that these snf mutants do not sufficiently activate AM signaling for the expression of these genes. SbtS expression was induced transiently in early stages of nodule formation; thus, 4 weeks after germination might be too late to observe RNS-related expression of $S b t S$ (Kistner et al. 2005; Takeda et al. 2009). However, SbtS not only is induced in early stages of AM but also is detected several weeks after inoculation with $G$. intraradices (Takeda et al. 2009). Therefore, SbtS induction should be observed if the AM signaling pathway was active in $s n f$ mutants.

\section{Deletion and substitution analysis of $S b t M 1$ promoter.}

The common SYM genes are required for SbtMl induction (Fig. 1A) (Kistner et al. 2005) but the deregulation of CCaMK, the central regulatory hub of the common SYM pathway (Hayashi et al. 2010; Madsen et al. 2010), is not sufficient for its expression (Fig. 1B). These observations indicated the existence of additional regulatory circuits mediating SbtMI induction during AM. However, AM-specific trans- and cis-active elements downstream of the common $S Y M$ pathway are unknown. We searched for AM-responsive cis-acting elements within the SbtM1 promoter in order to analyze the SbtMI activation mechanism and increase our understanding of AMspecific gene regulation.

The SbtM1 promoter $(-688$ to $+7 \mathrm{bp}$, start codon +1 to +3 bp) was fused to the GUS reporter gene, and the promoter activity during AM was monitored (Supplementary Fig. S1) (Takeda et al. 2009). In noninoculated transgenic roots carrying $S b t M 1_{p r o}: G U S$, blue staining was observed around the vascular bundle. After inoculation with $G$. intraradices, the GUS staining around the vascular bundle disappeared and distinct GUS staining was observed in epidermal and cortical cells around internal fungal structures in the host roots (Takeda et al. 2009). A characteristic patchy staining pattern was often observed 1 week after inoculation (wai) and the stained regions corresponded to the entrance points of fungal hyphae into the host root (Takeda et al. 2009). In later stages of AM development, hyphae colonize the host root and form AM structures such as arbuscules and vesicles. The SbtM1 promoter activity increased in cortical cells around internal fungal hyphae at 2 wai. Especially, arbuscule-containing cells showed stronger GUS staining than neighboring cells.

We used these two distinct patterns, patchy staining around fungal entry points and strong staining in arbuscule-containing cells, as criteria for the qualitative evaluation of AM responsiveness of the SbtM1 promoter in the following experiments. We observed differences in staining intensities between individual hairy roots. Possible explanations for these variations are position effects of the transgene, its copy number, the age of the roots, and AM infection levels. Therefore, a quantitative analysis of GUS activity in extracts of transformed roots appeared inappropriate to determine the AM responsiveness of the promoters analyzed.

A deletion and a substitution series of the SbtMl promoter was fused to the GUS reporter and promoter activity was analyzed in response to AM inoculation (Fig. 2). The analysis of the deletion series revealed one cis region between -161 and -109 bp upstream of the ATG of the translation start codon $(+1$ to $+3 \mathrm{bp}$ ) (Fig. 2A; Supplementary Fig. S2). The SbtM1 promoter fusions that retained at least $-161 \mathrm{bp}$ resulted in a clear 
patchy staining and a strong staining in arbuscule-containing cells. However, SbtM1 $1_{\text {prolog: }}$ GUS roots showed reduced or no GUS activity compared with $S b t M 1_{\text {prol61 }}$ :GUS roots. To narrow down the AM-response region, we tested seven constructs, each carrying one substituted stretch of 7 or $8 \mathrm{bp}(\mathrm{A} / \mathrm{C}$ and $\mathrm{T} / \mathrm{G}$ ) covering the region between $-158 \mathrm{bp}$ and $-109 \mathrm{bp}$ in the context of the $S b t M 1_{\text {prol61: }}$ GUS fusion construct (Fig. 2B). Two substituted $S b t M 1_{\text {prol61 }}: G U S$ constructs, one between -158 and -152 bp (SbtM1 $1_{\text {prol61sub158-152) and another between }-151 \text { and }}$

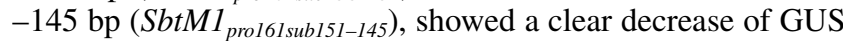
activity and no AM-induced GUS staining pattern (data not shown). This substitution analysis revealed that the region between -161 and $-145 \mathrm{bp}$ (region I) is required for AM-induced activation of the -161-bp SbtM1 promoter (Fig. 3A). However, when a substitution between -158 and -152 bp was tested in the context of a longer promoter of 688 bp (SbtM1 $1_{\text {pro688subl58-152: }}$ $G U S$ ), the typical AM-induced GUS staining pattern was observed, indicating the existence of additional AM-responsive cis elements in this promoter. In order to determine their positions, an SbtMl promoter deletion series carrying the substitu-

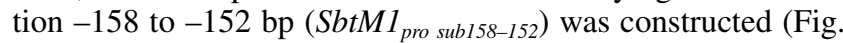
2C; Supplementary Fig. S3). In this deletion analysis, roots

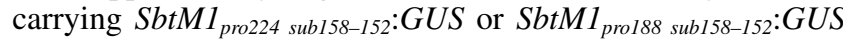
displayed no AM-specific staining, whereas roots transformed with the $S b t M 1_{\text {pro268 sub158-152:GUS construct showed a clear }}$ AM-induced GUS pattern. These results point to a second AM-responsive cis region between -268 and -225 bp. To narrow down this second cis region, a series of five constructs was tested, each carrying a 10-bp-long stretch of A/C and T/G base substitutions in the context of SbtM1 pro308 sub158-152:GUS (Fig. 2D). Roots carrying the $S b t M 1_{\text {pro308 sub158-152 sub248-239:GUS }}$ transgene showed the distinct AM-response staining.

However, from this series, the promoter constructs substituted between -268 and -259 bp (SbtM1 $1_{\text {pro308sub158-152sub268-259) }}$ and between -258 and -249 bp (SbtM1 pro308sub158-152sub258-249) $^{-25}$ did not induce the AM-specific GUS staining pattern and exhibited a clear decrease of GUS activity. A slight decrease of GUS staining was also observed in the construct substituted between -238 and -229 bp (data not shown). These results revealed two additional regions, one between -268 and $-249 \mathrm{bp}$ (region II) and the second between -238 and -229 bp (region III), that were important for AM responsiveness (Fig. 3A).

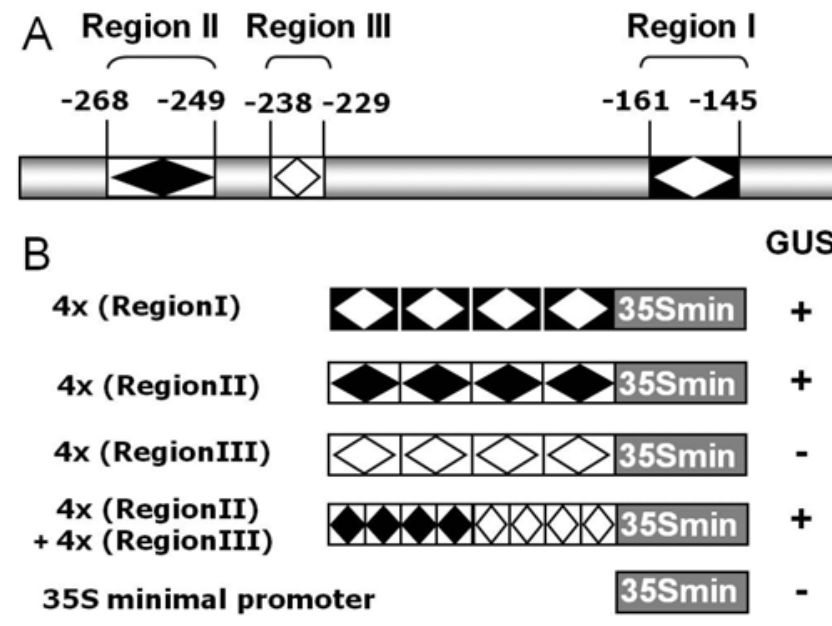

Fig. 3. Analysis of arbuscular mycorrhiza (AM)-response cis regions using a $35 \mathrm{~S}$ minimal promoter. A, Two predicted AM-response cis regions in the SbtM1 promoter, region I and region II, were described. B, Four tandem repeats of the predicted AM-response cis regions were fused to a $35 \mathrm{~S}$ minimal promoter $\beta$-glucuronidase (GUS) construct. AM-induced GUS activity in transgenic roots carrying the constructs was observed 1 and 2 weeks after AM inoculation.
We tested the relevance of the identified regions in the context of the longer $(688 \mathrm{bp})$ promoter. Constructs in which region I and II were substituted, such as $S b t M 1_{\text {pro688 sub158-152 }}$ sub268-259:GUS and SbtM1 pro688 sub158-152sub258-249: $G U S$, caused loss of the AM-induced GUS staining. Interestingly, a construct in which region I and III were substituted ( $S b t M 1_{\text {pro688 subl58-152 }}$ sub238-229:GUS) still conferred AM responsiveness (data not shown), suggesting that region II can compensate the mutation of region III in the context of the longer promoter and that the substitution of region III did not affect the AM responsiveness of region II. Importantly, this analysis did not reveal any other elements within the -688 -bp SbtM1 promoter region that are sufficient for AM responsiveness.

\section{Analysis of AM-response cis regions in the context of the $35 \mathrm{~S}$ minimal promoter.}

The deletion and substitution analysis revealed that the two cis-acting regions I and II in the SbtMI promoter are necessary for AM responsiveness while region III had a contributing effect. To test whether these cis regions are sufficient for AMinduced gene expression, they were fused as tandem repeats to a 46-bp-long $35 \mathrm{~S}$ minimal promoter $\left(35 \operatorname{Smin}_{\text {pro }}: G U S\right)$ and their response to AM inoculation was tested (Fig. 3). Transformed roots carrying $35 \operatorname{Smin}_{p r o}$ :GUS showed background staining around the vascular bundle. Weak staining was sometimes observed in cortical cells but it was not correlated with AM colonization (Supplementary Fig. S4A and B). SbtM1 pro(-161 to -145)x4: $35 \operatorname{Smin}_{\text {pro }}$ :GUS and $S b t M 1_{\text {pro(-268 to }-249) \times 4}: 35 \operatorname{Smin}_{p r o}$ :GUS roots showed a staining in epidermal cells and cortical cells around fungal hyphae similar to $S b t M 1_{p r o}: G U S$ (Fig. 3B). The arbuscule-containing cells showed strong GUS staining in these transformed roots. These results indicate that either region I or II is sufficient to induce GUS activity in response to AM infection.

In contrast, transgenic roots carrying $S b t M 1_{\text {pro }(-238 \text { to }-229) \times 4}$ : $35 \operatorname{Smin}_{\text {pro }}: G U S$ did not show an AM-induced GUS staining pattern and the staining was almost the same as that observed in control roots carrying $35 \operatorname{Smin}_{\text {pro }}: G U S$. Furthermore, we tested the $S b t M 1_{\text {pro(-268 to }-249) \times 4+(-238 \text { to }-229) \times 4}: 35 \operatorname{Smin}_{\text {pro }}$ :GUS construct (Fig. 3). Hairy roots expressing this construct were stained like

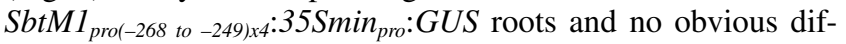
ferences were observed.

\section{Regions I and II represent novel AM-response regions.}

Region I and II sequences did not show significant similarity to known symbiosis-related cis elements from symbiosisinduced genes (Andriankaja et al. 2007; Boisson-Dernier et al. 2005; Fehlberg et al. 2005; Frenzel et al. 2006; Stougaard et al. 1987) (data not shown). Interrogation of plant promoter motif
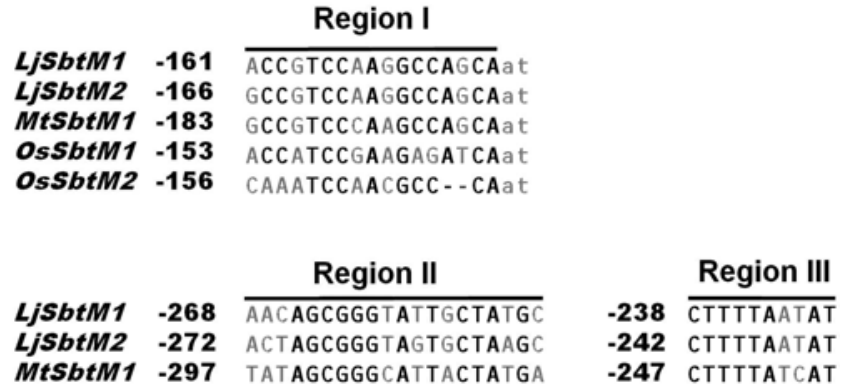

Fig. 4. Comparison of arbuscular mycorrhiza (AM)-response cis regions in AM-induced subtilases. Alignment of AM-response cis regions of SbtM1 with corresponding promoter sequences of SbtM2 and AM-induced subtilases in Medicago truncatula (MtSbtM1) and rice (OsSbtM1 and OsSbtM2). Black letters indicate conserved nucleotides in these promoter sequences. 
databases such as Place and plantCARE identified several plant cis elements with identical sequences. The sequence of CCGTCC in region I (Fig. 4) matched an elicitor response element called Box A identified within the PAL promoter of parsley (Petroselinum crispum) through in vivo foot printing (Logemann et al. 1995). The same sequence in region I was also identical to a cis element related to histone gene expression in maize (Brignon and Chaubet 1993). The sequence AGCGGG in region II conserved in the LjSbtM1, LjSbtM2, and MtSbtM1 promoters (Fig. 4) was found in a cinnamoylCoA reductase gene promoter required for vascular expression in Eucalyptus gunnii (Lacombe et al. 2000). In addition, another cis element, the carbohydrate metabolite signal responsive element (TGGACGG) (Morikami et al. 2005), was found on the negative strand of region I. Regions I and II contained sequence motifs related to previously identified cis elements but AM-responsiveness of these elements has not been reported thus far. Therefore, we conclude that regions I and II are novel AM-response regions.

\section{Conservation of AM-responsive cis regions in predicted SbtM1 orthologs across angiosperms.}

The AM-response cis sequences were found in the promoters of AM-induced subtilases in other AM-forming plant species. The putative orthologue of SbtM1 in M. truncatula (AW584611, MtSbtM1) is induced during AM (Liu et al. 2003; Takeda et al. 2009). Sequences with high similarity to regions I and II were found in its promoter (Fig. 4). Likewise, a sequence similar to region I was also detected in the promoter of an SbtM1 homolog from rice, OsSbtM1, that is induced during AM (called OsAM21 by Guimil and associates [2005]). OsSbtM1 occurs in a gene cluster and the paralog in the same cluster, named OsSbtM2, also carries a sequence similar to region I in a similar position of the promoter (Fig. 4).

\section{Regions I and II are present}

\section{in other AM regulated legume genes.}

Two other subtilase genes within the SbtM cluster, SbtM2 and SbtM3, carry similar sequences in similar positions of their promoters. The promoter of the pseudogene SbtM2 included a completely conserved region I, whereas region II showed only two mismatches (Fig. 4). However, no increase of SbtM2 transcript was observed during AM by quantitative RTPCR analysis (Fig. 1A). In contrast, transgenic roots carrying SbtM2 $2_{\text {pro }}: G U S$ showed almost the same GUS staining pattern as $S b t M 1_{p r o}: G U S$ in response to AM fungi infection (Supplementary Fig. S5). This result indicates that these SbtM2 cis regions are fully functional and the absence of expression of

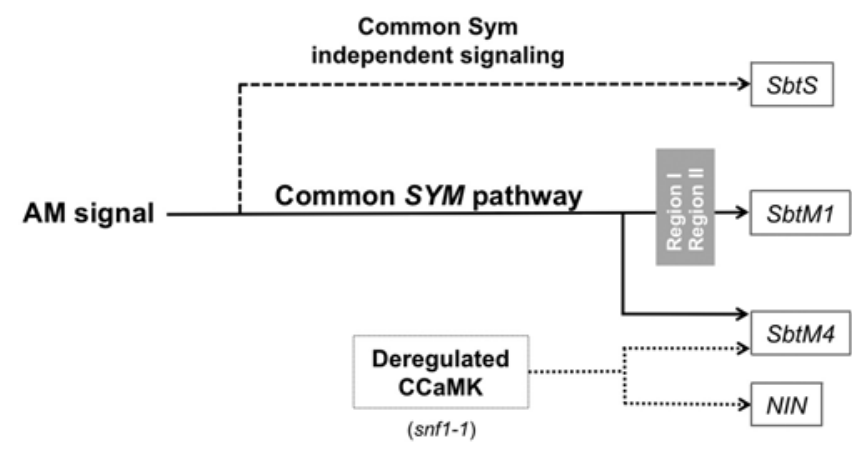

Fig. 5. Three symbiosis-related transcriptional response patters in Lotus japonicus as revealed by gene expression analysis. Deregulated CCaMK (snf1-1) activates SbtM4 and NIN. Arbuscular mycorrhiza (AM) fungal infection activates two parallel pathways: one common $S Y M$-gene-dependent pathway, leading to SbtM1 and SbtM4 activation, and a second, common $S Y M$-independent pathway sufficient for $S b t S$ activation. the endogenous SbtM2 may be the result of gene silencing of this pseudogene.

The expression pattern of SbtM3 was very similar to that of SbtM1 (Takeda et al. 2009; this work). Two sequences similar to region I were found in the SbtM3 promoter between -126 and -110 bp and between -112 and -96 bp (Supplementary Fig. S6A) but we could not find a sequence corresponding to region II in the SbtM3 promoter.

The phosphate transporter gene PT4 is expressed in arbuscule-containing cells and also carries a sequence with similarity to region I in the promoter between -215 and $-199 \mathrm{bp}$. However, these sequences were only weakly conserved and no sequences similar to region II could be identified within $1.0 \mathrm{~kb}$ upstream of the PT4 start codon. A deletion of the predicted region I sequence in the $P T 4$ promoter resulted in a decrease, but not a complete loss, of the specific induction in arbusculecontaining cells. This result revealed the presence of other cis elements in the remaining 200-bp promoter region, which were not analyzed further.

Among the AM-induced genes that carried sequences with similarity to region I or region II in the promoter, a group of genes was apparent that encode biosynthesis enzymes in the phenylpropane pathway - chalcone-flavonoid isomerase $(\mathrm{CHI}$, AP004250) and phenylalanine ammonia-lyase (PAL) - and in the subsequent flavonoid pathway, UDP-glucose:flavonoid glycosyltransferase (UGT; AP008049 and AP012031). They reside in gene clusters composed of four and five homologs (Supplementary Fig. S7B) (data not shown) and some of the family members were induced during AM (Deguchi et al. 2007; Guether et al. 2009). CHII (chr5.CM0260.370.nc) and UGT4 (chr4.CM0739.130.nd) contained sequences with similarity to region I. UGT4 was upregulated more than 60 times during AM development and some of the homologs in the same gene cluster were also upregulated. One of the $P A L$ genes, named PAL2 (DF093205, chr1.CM0033.830.nc) clustered with three other $P A L$ homologs and contained both region I- and region II-related sequences in the promoter, though their position in the promoter was opposite to the region I and region II in SbtMI. These results suggest that the same regulon may be involved in the AM induction of flavonoid synthesis genes and the AM-induced subtilases.

\section{DISCUSSION}

\section{Common symbiosis genes are necessary but CCaMK deregulation is not sufficient for AM-related gene activation.}

AM development is associated with the transcriptional regulation of hundreds of plant genes. This transcriptional reprogramming during AM comprises a set of core genes, including plant subtilases, that are consistently upregulated in plant species as diverse as legumes and rice (Guether et al. 2009; Guimil et al. 2005; Kistner et al. 2005; Liu et al. 2003). Mutations in common $S Y M$ genes almost completely abolish this transcriptional reprogramming in legumes (Kistner et al. 2005; Weidmann et al. 2004) and in rice (Gutjahr et al. 2008), demonstrating that the conceptual regulatory network defined by the common SYM genes is central to gene regulation in AM across angiosperms (Markmann and Parniske 2009; Parniske 2008). The subtilase gene SbtMl of L. japonicus is characterized by a particularly specific and strong AM responsiveness and is a member of a small group of symbiosis-induced subtilases. Interestingly, the expression patterns differ significantly among these genes. Whereas SbtM1 and SbtM3 are induced only during AM, SbtM4 and $S b t S$ are also induced in response to rhizobia (Takeda et al. 2009). Therefore, we have singled out this gene family to study transcriptional regulation during 
AM. We found that $S b t M 1$ and $S b t M 3$ were, like most other AM-regulated genes, not expressed in common sym mutants (Fig. 1A). On the other hand, SbtS expression was only reduced and retained a significant level of induction in common sym mutants. This result suggests that $S b t S$ is activated via signaling through a common $S Y M$ independent symbiotic or nonsymbiotic pathway (Fig. 5). The somewhat lower activation of $S b t S$ in the background of common sym mutants may be a consequence of reduced signaling input due to nonsuccessful fungal colonization. Alternatively, the common $S Y M$ pathway may synergistically contribute to $S b t S$ expression.

Evidence for common $S Y M$-independent gene regulation was previously obtained for AM-repressed genes in L. japonicus (Kistner et al. 2005). Furthermore, the expression of an AM-induced proteinase inhibitor gene in M. truncatula is independent of DMI2 (Kuhn et al. 2010), and ENOD11 is induced by diffusible factors from AM fungi through a common SYM (DMI1, DMI2, and DMI3)-independent pathway (Kosuta et al. 2003). Also, in rice, four AM-induced genes showed a reduced but significant level of induction in the background of common sym mutants, thus revealing an independent AM signaling pathway (Gutjahr et al. 2008, Mukherjee and Ané 2011). These consistent observations in a legume and in rice support the concept of at least two independent signaling pathways that contribute to the transcriptional reprogramming of the root during AM development.

The existence of common SYM-independent AM signaling was further supported by the analysis of gene activation in the $L$. japonicus snfl-1 and snf2-2 mutants that spontaneously form root nodules in the absence of rhizobia and encode constitutive active CCaMK and cytokinin receptor versions (Tirichine et al. 2006a; Tirichine et al. 2007). Consistent with the spontaneous nodule formation, nodulation-related genes like NIN and SbtM4 are transcriptionally activated (Fig. 1B) (Tirichine et al. 2006a; Tirichine et al. 2007), indicating that the snfl- and snf2-mediated signaling is sufficient for their activation. If the common SYM pathway was sufficient for AM gene regulation, one would expect an activation of the AM marker genes by the constitutively active snfl allele of CCaMK. However, snf1-1 and snf2-2 mutants did not show induction of SbtM1, SbtM3, or $S b t S$. These results indicate that the two snf alleles are sufficient for the activation of nodulation-related but not AM-related genes (Fig. 5). Thus, it appears the coordinated activity of the common SYM and other signaling pathways is required for mediating gene activation during AM. The existence of an AM-specific pathway would provide the necessary specificity, allowing appropriate responses to AM fungi or rhizobia as a result of common SYMrelated signaling.

\section{Identification of two novel AM-response cis sequences, one} of which is conserved in SbtM1 genes across angiosperms.

To obtain a molecular handle on regulatory circuits governing AM, we identified cis regions responsible for AM responsiveness of SbtM1. Our functional analysis of the SbtM1 promoter revealed that at least two apparent unrelated regulatory sequences are involved in its activation. However, because short sequences (CAGC and $\operatorname{AGCA}(\mathrm{AT})$ ) were conserved in the positive or negative strands of both regions I and II, we cannot exclude the possibility that the same transcription factors bind to regions I and II.

Both cis regions were conserved in the predicted SbtMI orthologue of M. truncatula, MtSbtM1 (Fig. 4). This suggests that MtSbtMI is regulated by the same cis elements and that orthologous trans-active factors exist in Medicago and Lotus spp. Two SbtM1 homologs in rice, OsSbtM1 and OsSbtM2, contain a sequence related to region I at a similar position in their promoters as in Lotus SbtMl, and OsSbtMl is induced during AM (Guimil et al. 2005). This conservation of region I reveals the presence of a common AM gene regulation system in AM plants as diversified as monocots and dicots.

We detected the presence of region I- or region II-related sequences in a subset of AM-responsive promoters of L. japonicus (data not shown). This result suggests that the two regions and their corresponding regulatory systems may contribute to the transcriptional reprogramming of the root during AM. However, the presence of region I- and II-like sequences is no proof that they are, indeed, responsible for the AM induction. Because the binding site of the corresponding trans factors is not yet known, the mismatches with the original region may or may not impair transcription factor binding. Furthermore, our analysis of the PT4 promoter clearly shows that other, yet-tobe identified elements are likewise able to mediate AM responsiveness.

\section{Modularity and diversity of AM-response cis elements.}

Regions I and II are, at least at the resolution of our assay, functionally redundant, because each one, on its own, was sufficient to confer AM responsiveness. This is in agreement with our sequence and expression analysis of the Lotus SbtM3 promoter, which confers a nearly identical expression pattern as the SbtM1 promoter (Takeda et al. 2009), although it contains a different set of cis elements, including two sequences with detectable similarity to region I but not to region II. We also found Lotus PT4 to be induced during AM. However, deletion analysis of the PT4 promoter suggested that additional or different cis elements govern its AM-specific expression.

The $c i s$ regions of $S b t M 1$ are distinct from previously identified AM-response cis regions from leghemoglobin in Vicia faba (Fehlberg et al. 2005) and lectin in M. truncatula (Frenzel et al. 2006). They also do not resemble known symbiosisresponse cis regions (Andriankaja et al. 2007; Boisson-Dernier et al. 2005; Stougaard et al. 1987). Because the SbtM1 cis regions do not share detectable sequence similarity, they are likely to be controlled by different symbiosis-responsive DNAbinding transcription factors.

Together, these data provide strong evidence that multiple cis elements exist that confer AM responsiveness. These elements can be found in different combinations and in different sequence variants. It is very likely that these different cis elements and combinations thereof contribute to the distinct spatiotemporal gene expression patterns observed for AM-responsive genes. Such a diversification of AM-responsive cis elements also implies that there are several distinct transcription factors involved in their binding and mediation of AM-induced gene expression.

Previous transcriptome analyses have identified a number of transcription factors whose expression was altered during RNS (Asamizu et al. 2005; El Yahyaoui et al. 2004; Lohar et al. 2006). Transcription factors of the GRAS and AP2/ERFfamilies were implicated in gene regulation during RNS (Andriankaja et al. 2007; Heckmann et al. 2006; Middleton et al. 2007). Although induction of transcription factor genes was also observed during AM (Guether et al. 2009; Guimil et al. 2005; Liu et al. 2003), genetic approaches to elucidate their role in AM transcriptional regulation have not been reported. Our promoter analysis facilitates the isolation of DNA-binding transcription factors as a next step in the investigation of the signaling pathways controlling gene activation during AM.

\section{MATERIALS AND METHODS}

\section{Biological material.}

Sterilized seed of L. japonicus Gifu B-129 and symbiotic mutants were placed on $0.8 \%$ bactoagar plates and grown in a 
growth chamber $\left(24^{\circ} \mathrm{C}, 16 \mathrm{~h}\right.$ of light and $8 \mathrm{~h}$ of darkness). The AM fungus $G$. intraradices (BEG 195) was proliferated in the chive nurse-pot system (Demchenko et al. 2004), which was used for AM inoculation of transgenic plants after removal of the chive plants. The snf1-1 and snf2-2 mutants were grown in sterilized soil for 4 weeks and checked for formation of spontaneous nodules and gene induction.

\section{Real time RT-PCR.}

Total RNA was extracted from roots using the NucleoSpin plant RNA extraction kit (Macherey-Nagel, Düren, Germany). RT and quantitative real-time PCR were performed using SuperScript III Platinum Two-Step qPCR kit with SYBR Green (Invitrogen, Darmstadt, Germany) and an iCycler (Bio-Rad, Munich) according to the manufacturer's instructions. The cDNA was synthesized from 50 ng of total RNA in a $20-\mu l$ RT reaction mixture and $1 \mu \mathrm{l}$ of the RT product was added to a total $20-\mu$ real-time PCR reaction mixture with $50 \mathrm{nM}$ fluorescein (Invitrogen) as a reference dye. Real-time PCR primer sets and thermal cycler condition described by Takeda and associates (2009) were used, and constitutively expressed gene elongation factor $1 \alpha$ and ubiquitin were used for reference.

Transcript expression levels were calculated using the PCR efficiency and normalized with the transcription level of ubiquitin. At least three biologically independent experiments were performed and the data were used to calculate average values and standard deviations.

\section{Construction of promoter GUS fusions.}

All primer sets to construct the fusion vectors were listed in Supplementary Table S1. For the SbtM1 promoter deletion series or the SbtM2 promoter GUS fusions, $S b t M$ promoter regions were amplified with PCR and the amplicons were cloned into pENTR D/TOPO (pENTR D/TOPO cloning kit; Invitrogen). For the substitution series, the entry clones of the SbtMI promoter deletion series were amplified using primers containing substitutions, and the amplicon containing the substituted SbtM1 promoter and the cloning vector was self-ligated with T4 DNA ligase (NEB). The inserts from the resulting entry clones were transferred into the promoter GUS fusion vector pKGWFS7 (Karimi et al. 2002) using in vitro site-directed recombination (LR reaction kit; Invitrogen).

The $35 \mathrm{~S}$ minimal promoter fusion vector was constructed on the basis of pCAMBIA1305. The Cauliflower mosaic virus $35 \mathrm{~S}$ promoter was removed except for $46 \mathrm{bp}$, leaving a minimal promoter region, and EcoRI and BamHI restriction sites were introduced $5^{\prime}$ of the $35 \mathrm{~S}$ minimal promoter. Oligonucleo-

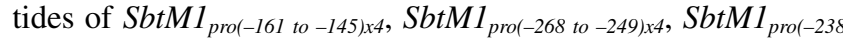
to -229)x4, or SbtM1 $1_{\text {pro(-268 to }-249) \times 4+(-238 \text { to }-229) \times 4}$ containing EcoRI and BamHI cohesive ends were cloned into the EcoRI and BamHI site of the $35 \mathrm{~S}$ minimal promoter vector.

\section{Transgenic hairy root induction by Agrobacterium rhizogenes.}

Agrobacterium rhizogenes AR1193, carrying the promoter fusion constructs, was used to transform L. japonicus via hairy root transformation as described by Takeda and associates (2009).

\section{Root inoculation, staining, and evaluation of AM responsiveness.}

Composite plants with 1 - to 2-cm-long transgenic hairy roots were inoculated with $G$. intraradices using the chive nurse-pot system (Demchenko et al. 2004) and cultivated in a growth chamber $\left(24^{\circ} \mathrm{C}, 16 \mathrm{~h}\right.$ of light, and $8 \mathrm{~h}$ of darkness). One or two weeks after inoculation, the roots were washed with water and stained with GUS-staining buffer (5-bromo-4-chloro-
3-indolyl-D-glucuronic acid at $0.5 \mathrm{mg} / \mathrm{ml}, 100 \mathrm{mM}$ phosphate buffer [pH 7.0], $100 \mathrm{mM}$ EDTA, $0.5 \mathrm{mM} \mathrm{K}_{4}\left[\mathrm{Fe}(\mathrm{CN})_{6}\right], 0.5$ $\mathrm{mM} \mathrm{K}_{3}\left[\mathrm{Fe}(\mathrm{CN})_{6}\right]$, and $0.1 \%$ Triton $\left.\mathrm{X}-100\right)$ for 3 to $6 \mathrm{~h}$ at $37^{\circ} \mathrm{C}$. Fungal structures were stained with wheat germ agglutinin (WGA) Alexa Fluor 488 (Invitrogen) as described by Harrison and associates (2002).

In all, 8 to 10 transgenic root systems per construct in one experiment were stained and observed using a stereoscopic microscope (MZ 16 FA; Leica, Wetzlar, Germany) or an inverted microscope (DMI6000B; Leica) to judge whether the stained roots exhibited the AM-specific staining pattern. Promoter constructs were scored AM responsive if a patchy staining around fungal entry points and strong staining in arbuscule-containing cells were observed. At least three independent experiments were conducted for one construct. Fluorescence microscopy of WGA Alexa Fluor 488 was performed using an inverted microscope (DMI6000B; Leica) equipped with $\times 40$ dry (numerical aperture [N.A.] 0.6) or $\times 63$ water immersion (N.A. 1.2) objectives.

\section{ACKNOWLEDGMENTS}

Mutant seed were kindly provided by J. Stougaard, K. Szczyglowski, and J. Webb. This work was supported by the German Research Foundation. N. Takeda was supported by a Postdoctoral Fellowship by the Japan Society for the Promotion of Science.

\section{LITERATURE CITED}

Akiyama, K., Matsuzaki, K., and Hayashi, H. 2005. Plant sesquiterpenes induce hyphal branching in arbuscular mycorrhizal fungi. Nature 435:824-827.

Andriankaja, A., Boisson-Dernier, A., Frances, L., Sauviac, L., Jauneau, A., Barker, D. G., and de Carvalho-Niebel, F. 2007. AP2-ERF transcription factors mediate Nod factor dependent Mt ENOD11 activation in root hairs via a novel cis-regulatory motif. Plant Cell 19:2866-2885.

Ane, J. M., Kiss, G. B., Riely, B. K., Penmetsa, R. V., Oldroyd, G. E., Ayax, C., Levy, J., Debelle, F., Baek, J. M., Kalo, P., Rosenberg, C., Roe, B. A., Long, S. R., Denarie, J., and Cook, D. R. 2004. Medicago truncatula DMI1 required for bacterial and fungal symbioses in legumes. Science 303:1364-1367.

Asamizu, E., Nakamura, Y., Sato, S., and Tabata, S. 2005. Comparison of the transcript profiles from the root and the nodulating root of the model legume Lotus japonicus by serial analysis of gene expression. Mol. Plant-Microbe Interact. 18:487-498.

Bago, B., Pfeffer, P. E., Abubaker, J., Jun, J., Allen, J. W., Brouillette, J., Douds, D. D., Lammers, P. J., and Shachar-Hill, Y. 2003. Carbon export from arbuscular mycorrhizal roots involves the translocation of carbohydrate as well as lipid. Plant Physiol. 131:1496-1507.

Banba, M., Gutjahr, C., Miyao, A., Hirochika, H., Paszkowski, U., Kouchi, H., and Imaizumi-Anraku, H. 2008. Divergence of evolutionary ways among common sym genes: CASTOR and CCaMK show functional conservation between two symbiosis systems and constitute the root of a common signaling pathway. Plant Cell Physiol. 49:1659-1671.

Besserer, A., Puech-Pages, V., Kiefer, P., Gomez-Roldan, V., Jauneau, A., Roy, S., Portais, J.C., Roux, C., Becard, G., and Sejalon-Delmas, N. 2006. Strigolactones stimulate arbuscular mycorrhizal fungi by activating mitochondria. PLoS Biol. 4:e226. Published online.

Boisson-Dernier, A., Andriankaja, A., Chabaud, M., Niebel, A., Journet, E. P., Barker, D. G., and de Carvalho-Niebel, F. 2005. MtENOD11 gene activation during rhizobial infection and mycorrhizal arbuscule development requires a common AT-rich-containing regulatory sequence. Mol. Plant-Microbe Interact. 18:1269-1276.

Bonfante, P., and Perotto, S. 1995. Tansley Review No. 82. Strategies of arbuscular mycorrhizal fungi when infecting host plants. New Phytol. 130:3-21.

Brignon, P., and Chaubet, N. 1993. Constitutive and cell-division-inducible protein-DNA interactions in two maize histone gene promoters. Plant J. 4:445-457.

Chabaud, M., Genre, A., Sieberer, B. J., Faccio, A., Fournier, J., Novero, M., Barker, D. G., and Bonfante, P. 2011. Arbuscular mycorrhizal hyphopodia and germinated spore exudates trigger $\mathrm{Ca}^{2+}$ spiking in the legume and nonlegume root epidermis. New Phytol. 189:347-355.

Chen, C., Gao, M., Liu, J., and Zhu, H. 2007. Fungal symbiosis in rice requires an ortholog of a legume common symbiosis gene encoding a $\mathrm{Ca}^{2+} /$ 
calmodulin-dependent protein kinase. Plant Physiol. 145:1619-1628.

Chen, C., Ane, J. M., and Zhu, H. 2008. OsIPD3, an ortholog of the Medicago truncatula DMI3 interacting protein IPD3, is required for mycorrhizal symbiosis in rice. New Phytol. 180:311-315.

Deguchi, Y., Banba, M., Shimoda, Y., Chechetka, S. A., Suzuri, R., Okusako, Y., Ooki, Y., Toyokura, K., Suzuki, A., Uchiumi, T., Higashi, S., Abe, M., Kouchi, H., Izui, K., and Hata, S. 2007. Transcriptome profiling of Lotus japonicus roots during arbuscular mycorrhiza development and comparison with that of nodulation. DNA Res. 14:117-133.

Demchenko, K., Winzer, T., Stougaard, J., Parniske, M., and Pawlowski, K. 2004. Distinct roles of Lotus japonicus SYMRK and SYM15 in root colonization and arbuscule formation. New Phytol. 163:381-392.

Drissner, D., Kunze, G., Callewaert, N., Gehrig, P., Tamasloukht, M., Boller, T., Felix, G., Amrhein, N., and Bucher, M. 2007. Lyso-phosphatidylcholine is a signal in the arbuscular mycorrhizal symbiosis. Science 318:265-268.

El Yahyaoui, F., Kuster, H., Ben Amor, B., Hohnjec, N., Puhler, A. Becker, A., Gouzy, J., Vernie, T., Gough, C., Niebel, A., Godiard, L., and Gamas, P. 2004. Expression profiling in Medicago truncatula identifies more than 750 genes differentially expressed during nodulation, including many potential regulators of the symbiotic program. Plant Physiol. 136:3159-3176.

Endre, G., Kereszt, A., Kevei, Z., Mihacea, S., Kalo, P., and Kiss, G. B. 2002. A receptor kinase gene regulating symbiotic nodule development. Nature 417:962-966.

Fehlberg, V., Vieweg, M. F., Dohmann, E. M., Hohnjec, N., Puhler, A., Perlick, A. M., and Kuster, H. 2005. The promoter of the leghaemoglobin gene VfLb29: functional analysis and identification of modules necessary for its activation in the infected cells of root nodules and in the arbuscule-containing cells of mycorrhizal roots. J. Exp. Bot. 56:799-806.

Floss, D. S., Hause, B., Lange, P. R., Kuster, H., Strack, D., and Walter, M. H. 2008. Knock-down of the MEP pathway isogene 1-deoxy-D-xylulose 5-phosphate synthase 2 inhibits formation of arbuscular mycorrhizainduced apocarotenoids, and abolishes normal expression of mycorrhiza-specific plant marker genes. Plant J. 56:86-100.

Frenzel, A., Tiller, N., Hause, B., and Krajinski, F. 2006. The conserved arbuscular mycorrhiza-specific transcription of the secretory lectin MtLec5 is mediated by a short upstream sequence containing specific protein binding sites. Planta 224:792-800.

Genre, A., Chabaud, M., Timmers, T., Bonfante, P., and Barker, D. G. 2005. Arbuscular mycorrhizal fungi elicit a novel intracellular apparatus in Medicago truncatula root epidermal cells before infection. Plant Cell 17:3489-3499.

Genre, A., Chabaud, M., Faccio, A., Barker, D. G., and Bonfante, P. 2008. Prepenetration apparatus assembly precedes and predicts the colonization patterns of arbuscular mycorrhizal fungi within the root cortex of both Medicago truncatula and Daucus carota. Plant Cell 20:1407-1420.

Gianinazzi-Pearson, V. 1996. Plant cell responses to arbuscular mycorrhizal fungi: getting to the roots of the symbiosis. Plant Cell 8:1871-1883.

Groth, M., Takeda, N., Perry, J., Uchida, H., Dräxl, S., Brachmann, A., Sato, S., Tabata, S., Kawaguchi, M., Wang, T. L., and Parniske, M. 2010. NENA, a Lotus japonicus homolog of Sec13, is required for rhizodermal infection by arbuscular mycorrhiza fungi and rhizobia but dispensable for cortical endosymbiotic development. Plant Cell 22:2509-2526.

Guether, M., Balestrini, R., Hannah, M., He, J., Udvardi, M. K., and Bonfante, P. 2009. Genome-wide reprogramming of regulatory networks, transport, cell wall and membrane biogenesis during arbuscular mycorrhizal symbiosis in Lotus japonicus. New Phytol. 182:200-212.

Guimil, S., Chang, H. S., Zhu, T., Sesma, A., Osbourn, A., Roux, C., Ioannidis, V., Oakeley, E. J., Docquier, M., Descombes, P., Briggs, S. P., and Paszkowski, U. 2005. Comparative transcriptomics of rice reveals an ancient pattern of response to microbial colonization. Proc. Natl. Acad. Sci. U.S.A. 102:8066-8070.

Gutjahr, C., Banba, M., Croset, V., An, K., Miyao, A., An, G., Hirochika, H., Imaizumi-Anraku, H., and Paszkowski, U. 2008. Arbuscular mycorrhiza-specific signaling in rice transcends the common symbiosis signaling pathway. Plant Cell 20:2989-3005

Harrison, M. J. 2005. Signaling in the arbuscular mycorrhizal symbiosis. Annu. Rev. Microbiol. 59:19-42.

Harrison, M. J., Dewbre, G. R., and Liu, J. 2002. A phosphate transporter from Medicago truncatula involved in the acquisition of phosphate released by arbuscular mycorrhizal fungi. Plant Cell 14:2413-2429.

Hayashi, T., Banba, M., Shimoda, Y., Kouchi, H., Hayashi, M., and Imaizumi-Anraku, H. 2010. A dominant function of CCaMK in intracellular accommodation of bacterial and fungal endosymbionts. Plant $\mathrm{J}$. 63:141-154.

Heckmann, A. B., Lombardo, F., Miwa, H., Perry, J. A., Bunnewell, S., Parniske, M., Wang, T. L., and Downie, J. A. 2006. Lotus japonicus nodulation requires two GRAS domain regulators, one of which is functionally conserved in a non-legume. Plant Physiol. 142:1739-1750. Imaizumi-Anraku, H., Takeda, N., Charpentier, M., Perry, J., Miwa, H., Umehara, Y., Kouchi, H., Murakami, Y., Mulder, L., Vickers, K., Pike, J., Downie, J. A., Wang, T., Sato, S., Asamizu, E., Tabata, S., Yoshikawa, M., Murooka, Y., Wu, G. J., Kawaguchi, M., Kawasaki, S. Parniske, M., and Hayashi, M. 2005. Plastid proteins crucial for symbiotic fungal and bacterial entry into plant roots. Nature 433:527-531.

Javot, H., Penmetsa, R.V., Terzaghi, N., Cook, D. R., and Harrison, M. J. 2007. A Medicago truncatula phosphate transporter indispensable for the arbuscular mycorrhizal symbiosis. Proc. Natl. Acad. Sci. U.S.A. 104:1720-1725

Karimi, M., Inze, D., and Depicker, A. 2002. GATEWAY vectors for Agrobacterium-mediated plant transformation. Trends Plant Sci. 7:193-195.

Kistner, C., and Parniske, M. 2002. Evolution of signal transduction in intracellular symbiosis. Trends Plant Sci. 7:511-518.

Kistner, C., Winzer, T., Pitzschke, A., Mulder, L., Sato, S., Kaneko, T., Tabata, S., Sandal, N., Stougaard, J., Webb, K. J., Szczyglowski, K., and Parniske, M. 2005. Seven Lotus japonicus genes required for transcriptional reprogramming of the root during fungal and bacterial symbiosis. Plant Cell 17:2217-2229.

Kosuta, S., Chabaud, M., Lougnon, G., Gough, C., Denarie, J., Barker, D. G., and Becard, G. 2003. A diffusible factor from arbuscular mycorrhizal fungi induces symbiosis-specific MtENOD11 expression in roots of Medicago truncatula. Plant Physiol. 131:952-962.

Kosuta, S., Hazledine, S., Sun, J., Miwa, H., Morris, R. J., Downie, J. A. and Oldroyd, G. E. 2008. Differential and chaotic calcium signatures in the symbiosis signaling pathway of legumes. Proc. Natl. Acad. Sci. U.S.A. 105:9823-9828.

Kuhn, H., Kuster, H., and Requena, N. 2010. Membrane steroid-binding protein 1 induced by a diffusible fungal signal is critical for mycorrhization in Medicago truncatula. New Phytol. 185:716-733.

Lacombe, E., Van Doorsselaere, J., Boerjan, W., Boudet, A. M., and Grima-Pettenati, J. 2000. Characterization of cis-elements required for vascular expression of the cinnamoyl $\mathrm{CoA}$ reductase gene and for protein-DNA complex formation. Plant J. 23:663-676.

Levy, J., Bres, C., Geurts, R., Chalhoub, B., Kulikova, O., Duc, G., Journet, E. P., Ane, J. M., Lauber, E., Bisseling, T., Denarie, J., Rosenberg, C., and Debelle, F. 2004. A putative Ca2+ and calmodulindependent protein kinase required for bacterial and fungal symbioses. Science 303:1361-1364.

Liu, J., Blaylock, L. A., Endre, G., Cho, J., Town, C. D., VandenBosch, K. A., and Harrison, M. J. 2003. Transcript profiling coupled with spatial expression analyses reveals genes involved in distinct developmental stages of an arbuscular mycorrhizal symbiosis. Plant Cell 15:21062123

Logemann, E., Parniske, M., and Hahlbrock, K. 1995. Modes of expression and common structural features of the complete phenylalanine ammonia-lyase gene family in parsley. Proc. Natl. Acad. Sci. U.S.A. 92:5905-5909.

Lohar, D. P., Sharopova, N., Endre, G., Penuela, S., Samac, D., Town, C., Silverstein, K. A., and VandenBosch, K. A. 2006. Transcript analysis of early nodulation events in Medicago truncatula. Plant Physiol. 140:221-234.

Madsen, L. H., Tirichine, L., Jurkiewicz, A., Sullivan, J. T., Heckmann, A. B., Bek, A. S., Ronson, C. W., James, E. K., and Stougaard, J. 2010. The molecular network governing nodule organogenesis and infection in the model legume Lotus japonicus. Nat. Commun. 1:1-12.

Maeda, D., Ashida, K., Iguchi, K., Chechetka, S.A., Hijikata, A., Okusako, Y., Deguchi, Y., Izui, K., and Hata, S. 2006. Knockdown of an arbuscular mycorrhiza-inducible phosphate transporter gene of Lotus japonicus suppresses mutualistic symbiosis. Plant Cell Physiol. 47:807-817.

Markmann, K., and Parniske, M. 2009. Evolution of root endosymbiosis with bacteria: how novel are nodules? Trends Plant Sci. 14:77-86.

Markmann, K., Giczey, G., and Parniske, M. 2008. Functional adaptation of a plant receptor-kinase paved the way for the evolution of intracellular root symbioses with bacteria. PLoS Biol. 6:e68.

Middleton, P. H., Jakab, J., Penmetsa, R. V., Starker, C. G., Doll, J., Kalo, P., Prabhu, R., Marsh, J. F., Mitra, R. M., Kereszt, A., Dudas, B. VandenBosch, K., Long, S. R., Cook, D. R., Kiss, G. B., and Oldroyd, G. E. 2007. An ERF transcription factor in Medicago truncatula that is essential for Nod factor signal transduction. Plant Cell 19:1221-1234.

Morikami, A., Matsunaga, R., Tanaka, Y., Suzuki, S., Mano, S., and Nakamura, K. 2005. Two cis-acting regulatory elements are involved in the sucrose-inducible expression of the sporamin gene promoter from sweet potato in transgenic tobacco. Mol. Gen. Genet. 272:690-699.

Mukherjee, A., and Ane, J. M. 2011. Germinating spore exudates from arbuscular mycorrhizal fungi: molecular and developmental responses in plants and their regulation by ethylene. Mol. Plant-Microbe Interact. 24:260-270. 
Oldroyd, G. E., and Downie, J. A. 2008. Coordinating nodule morphogenesis with rhizobial infection in legumes. Annu. Rev. Plant Biol. 59:519-546.

Parniske, M. 2008. Arbuscular mycorrhiza: the mother of plant root endosymbioses. Nat. Rev. Microbiol. 6:763-775.

Rawlings, N. D., and Barrett, A. J. 1999. MEROPS: the peptidase database. Nucleic Acids Res. 27:325-331.

Schüssler, A., Schwarzott, D., and Walker, C. 2001. A new fungal phylum, the Glomeromycota: phylogeny and evolution. Mycol. Res. 105:14131421.

Siezen, R. J., and Leunissen, J. A. 1997. Subtilases: the superfamily of subtilisin-like serine proteases. Protein Sci. 6:501-523.

Stougaard, J., Sandal, N. N., Gron, A., Kuhle, A., and Marcker, K. A. 1987. 5' Analysis of the soybean leghaemoglobin $\operatorname{lbc}(3)$ gene: regulatory elements required for promoter activity and organ specificity. EMBO (Eur. Mol. Biol. Organ.) J. 6:3565-3569.

Stracke, S., Kistner, C., Yoshida, S., Mulder, L., Sato, S., Kaneko, T., Tabata, S., Sandal, N., Stougaard, J., Szczyglowski, K., and Parniske, M. 2002. A plant receptor-like kinase required for both bacterial and fungal symbiosis. Nature 417:959-962.

Takeda, N., Kistner, C., Kosuta, S., Winzer, T., Pitzschke, A., Groth, M., Sato, S., Kaneko, T., Tabata, S., and Parniske, M. 2007. Proteases in plant root symbiosis. Phytochemistry 68:111-121.

Takeda, N., Sato, S., Asamizu, E., Tabata, S., and Parniske, M. 2009. Apoplastic plant subtilases support arbuscular mycorrhiza development in Lotus japonicus. Plant J. 58:766-777.

Tirichine, L., Imaizumi-Anraku, H., Yoshida, S., Murakami, Y., Madsen, L. H., Miwa, H., Nakagawa, T., Sandal, N., Albrektsen, A. S., Kawaguchi, M., Downie, A., Sato, S., Tabata, S., Kouchi, H., Parniske, M., Kawasaki,
S., and Stougaard, J. 2006a. Deregulation of a $\mathrm{Ca}^{2+} /$ calmodulin-dependent kinase leads to spontaneous nodule development. Nature 441:11531156.

Tirichine, L., James, E. K., Sandal, N., and Stougaard, J. 2006b. Spontaneous root-nodule formation in the model legume Lotus japonicus: a novel class of mutants nodulates in the absence of rhizobia. Mol. PlantMicrobe Interact. 19:373-382.

Tirichine, L., Sandal, N., Madsen, L. H., Radutoiu, S., Albrektsen, A. S. Sato, S., Asamizu, E., Tabata, S., and Stougaard, J. 2007. A gain-offunction mutation in a cytokinin receptor triggers spontaneous root nodule organogenesis. Science 315:104-107.

Weidmann, S., Sanchez, L., Descombin, J., Chatagnier, O., Gianinazzi, S., and Gianinazzi-Pearson, V. 2004. Fungal elicitation of signal transduction-related plant genes precedes mycorrhiza establishment and requires the dmi3 gene in Medicago truncatula. Mol. Plant-Microbe Interact. 17:1385-1393.

Wegel, E., Schauser, L., Sandal, N., Stougaard, J., and Parniske, M. 1998. Mycorrhiza Mutants of Lotus japonicus define genetically independent steps during symbiotic infection. Mol. Plant-Microbe Interact. 11:933936.

Yano, K., Yoshida, S., Müller, J., Singh, S., Banba, M., Vickers, K. Markmann, K., White, C., Schuller, B., Sato, S., Asamizu, E., Tabata, S., Murooka, Y., Perry, J., Wang, T. L., Kawaguchi, M., ImaizumiAnraku, H., Hayashi, M., and Parniske, M. 2008. CYCLOPS, a mediator of symbiotic intracellular accommodation. Proc. Natl. Acad. Sci. U.S.A. 105:20540-20545.

Zhang Q., Blaylock L. A., and Harrison M. J. 2010. Two Medicago truncatula Half-ABC transporters are essential for arbuscule development in arbuscular mycorrhizal symbiosis. Plant Cell 22:1483-1497. 